University of Nebraska - Lincoln

DigitalCommons@University of Nebraska - Lincoln

U.S. Department of Veterans Affairs Staff

Publications

U.S. Department of Veterans Affairs

2008

\title{
The impact of apathy on glycemic control in diabetes: A cross- sectional study
}

Prasad R. Padala

University of Nebraska Medical Center, ppadala@unmc.edu

Cyrus V. Desouza

University of Nebraska Medical Center, cdesouza@unmc.edu

Stephanie Almeida

University of Nebraska Medical Center

Vijay Shivaswamy

University of Nebraska Medical Center, vshivaswamy@unmc.edu

Krishan Ariyarathna

Creighton University

See next page for additional authors

Follow this and additional works at: https://digitalcommons.unl.edu/veterans

Padala, Prasad R.; Desouza, Cyrus V.; Almeida, Stephanie; Shivaswamy, Vijay; Ariyarathna, Krishan; Rouse, Lance; Burke, William J.; and Petty, Frederick, "The impact of apathy on glycemic control in diabetes: A cross-sectional study" (2008). U.S. Department of Veterans Affairs Staff Publications. 62.

https://digitalcommons.unl.edu/veterans/62

This Article is brought to you for free and open access by the U.S. Department of Veterans Affairs at DigitalCommons@University of Nebraska - Lincoln. It has been accepted for inclusion in U.S. Department of Veterans Affairs Staff Publications by an authorized administrator of DigitalCommons@University of Nebraska - Lincoln. 


\section{Authors}

Prasad R. Padala, Cyrus V. Desouza, Stephanie Almeida, Vijay Shivaswamy, Krishan Ariyarathna, Lance Rouse, William J. Burke, and Frederick Petty 


\title{
The impact of apathy on glycemic control in diabetes: A cross-sectional study
}

\author{
Prasad R. Padala ${ }^{a, b}$, Cyrus V. Desouza ${ }^{a, b, *}$, Stephanie Almeida ${ }^{b}$, Vijay Shivaswamy ${ }^{b}$, \\ Krishan Ariyarathna ${ }^{c}$, Lance Rouse ${ }^{b}$, William J. Burke ${ }^{b}$, Frederick Petty ${ }^{a, c}$ \\ ${ }^{a}$ Veterans Affairs Medical Center, Omaha, NE, United States \\ ${ }^{\mathrm{b}}$ University of Nebraska Medical Center, Omaha, NE, United States \\ ${ }^{\mathrm{c}}$ Creighton University, Omaha, NE, United States
}

\section{A R T I C L E I N F O}

Article history:

Received 13 March 2007

Accepted 26 June 2007

Published on line 6 August 2007

\section{Keywords:}

Apathy

Diabetes mellitus

HbA1c

Glycemic control

\begin{abstract}
A B S T R A C T
Objective: Diabetes mellitus is a major public health problem with a prevalence of 6-7\%. Selfcare behaviors play a major role in the control of diabetes. Apathy is characterized by loss of initiative and motivation. Apathy may interfere with self-care behavior and glycemic control. The primary objective was to determine the prevalence of apathy in patients with diabetes. The secondary objective was to determine if there was an association between clinically significant apathy and factors that affect glycemic control.

Research design and methods: We conducted a cross-sectional study of 100 patients with diabetes who were assessed with the Apathy Evaluation Scale-Clinician version (AES-C), the Hamilton Depression Scale (HAM-D), and the Self-Care Inventory (SCI). For this study we defined clinically significant apathy as AES-C score of $>30$. We excluded patients with a HAM-D score of $>14(n=19)$ to avoid confounding from depression. T-tests were used to compare clinical characteristics between subjects with and without apathy. Multiple linear regression modeling was used to investigate the association between clinically significant apathy and factors that affect glycemic control.

Results: Fifty (61.7\% of 81$)$ patients had clinically significant apathy. Compared to the nonapathetic patients, those with apathy had a higher mean BMI $\left(30.5 \mathrm{~kg} / \mathrm{m}^{2}\right.$ versus $34.1 \mathrm{~kg} / \mathrm{m}^{2}$ $(p=0.03))$ and were less likely to adhere to an exercise plan $(p=0.01)$ or insulin regimen $(p=0.003)$. After adjustment for age, BMI, cholesterol, mild depression and the average SelfCare Index score, the mean $\mathrm{HbA1C}$ level was $0.66 \%$ greater for apathetic compared to nonapathetic subjects $(P=0.08)$.

Conclusion: Apathy is highly prevalent in patients with diabetes without depression. Apathy may have a negative impact on self-care behaviors and diabetes control.
\end{abstract}

Published by Elsevier Ireland Ltd.

\section{Introduction}

Diabetes mellitus is a major public health problem. The prevalence of diabetes in the general population in the USA is estimated to be $6-7 \%$ [1]. Prevalence rates of diabetes in the Department of Veterans Affairs (VA) health care system are estimated to be $19-20 \%$ [2]. The prevalence of diabetes in the VA Nebraska Western Iowa Healthcare System (NWIHCS) is

\footnotetext{
* Corresponding author at: Department of Internal Medicine (111), Omaha Veterans Affairs Medical Center, 4101 Woolworth Avenue, Omaha, NE 68105, United States. Tel.: +1 402346 8800x5506; fax: +1 4029775602.

E-mail address: cdesouza@unmc.edu (C.V. Desouza). 0168-8227/\$ - see front matter. Published by Elsevier Ireland Ltd.
} doi:10.1016/j.diabres.2007.06.012 
$20-22 \%$, which is three times the prevalence of diabetes in the general population [3].

The increasing prevalence of diabetes has led to an epidemic of cardiovascular and other complications such as blindness, end-stage renal disease, and lower limb amputations. Poor glycemic control contributes to the high incidence of these complications [4]. Diabetes is the fifth leading cause of death in the United States. Total cost of caring for diabetes including the direct medical, and indirect expenditures in 2002 were estimated at US $\$ 132$ billion [5]. Several trials have shown that controlling the blood sugars, LDL cholesterol and blood pressure of patients with diabetes reduce risk of these complications. Yet less than $10 \%$ of patients with diabetes have reached their goals in all three parameters [6]. There are several factors why patients with diabetes may not achieve the desired goals. Co-morbid mental illness has been found to increase this risk.

The presence of mental illness complicates the course of diabetes. Depression is known to be an independent risk factor for diabetes and increases the risk of developing diabetes by $23 \%$ in younger adults $[7,8]$. According to a meta-analysis conducted by de Groot et al. [9], there is a significant association between depression and several diabetes complications. Factors that may be responsible for this increased risk include obesity, smoking, unhealthy dietary habits and inadequate utilization of preventative and primary healthcare, and nonadherence to medications [8].

There are conflicting data concerning the impact of depression on glycemic control. Singh et al. showed in a Pima Indian population that $\mathrm{HbA1C}$ was significantly higher in depressed patients with diabetes when compared with those without depression [10]. Severity of depressive symptomatology has been linked to poorer adherence to dietary, and medication regimen, higher functional impairment and higher health care costs in patients with diabetes [11]. However in a large study of patients with diabetes, only a weak relationship between depression and HbA1c was found at baseline and the presence of depression did not prospectively predict change in glycemic control [12].

Apathy, rather than depression, may predict adherence to diabetic self-care and may have a closer link with glycemic control. Apathy, a common behavioral problem in chronic illnesses such as Parkinsonism, Multiple Sclerosis, Alzheimer's dementia, is a clinical syndrome that is distinct from depression $[13,14]$. Self-criticism and negative outlook of future are common emotions in depression, and are absent in apathetic individuals, who show a lack of concern instead which may have direct adverse impact on self-care of diabetes $[15,16]$. Apathy is characterized by loss of initiation and motivation, decreased social engagement, and emotional indifference [15]. Apathy manifests as poor persistence, lack of interest, blunted emotional response and lack of insight [16]. It is common and occurs alone and together with depression. Apathy has profound consequences on both patients and caregivers [17]. Patients with apathy were 3.2 times more likely to have impaired activities of daily living in a sample of patients with dementia [18].

The proper care of diabetes requires the patient to exercise regularly, adhere to recommended diet and food portions, keep food records, attend clinics regularly, check blood sugars frequently and take medications or insulin at correct times and dosages. The presence of apathy may keep patients from adhering to this regimen and following the self-care recommendations [19]. In one study self-reported apathy was shown to be one of the reasons why patients with diabetes did not return for a recommended eye examination [20].

However, to our knowledge, there are no studies looking at the prevalence of apathy in patients with diabetes. Further, no systematic study has addressed the impact of apathy on glycemic control and self-care behavior. Also, there are no studies to examine the effect of apathy on diabetes independent of depression. The primary objective of this study was to determine the prevalence of apathy in patients with diabetes. The secondary objective was to determine if there was an association between clinically significant apathy and factors that affect glycemic control.

\section{Research design and methods}

We conducted a cross-sectional study of 100 patients with diabetes mellitus enrolled in the Diabetes Clinic, Primary Care Clinic, Mental Health Clinic and the Recovery Center at the Omaha Veterans Affairs Medical Center (OVAMC). The institutional review board at OVAMC approved the study. Patients were approached by the research team members and invited to participate in the study and provided written consent before participating in the study.

Each patient participated in a 60-75 min semi-structured interview to complete the Apathy Evaluation Scale-Clinician version (AES-C) [21], the Hamilton Rating Scale for Depression17 item version $\left(H A M-D_{17}\right)$ [22], and The Self-Care Inventory (SCI) [23]. Nineteen patients scoring $>14$ on the HAM-D were excluded from analysis to help avoid confounding from the presence of clinically significant depression. Data from the remaining 81 patients were analyzed.

Demographic and laboratory data were also collected from the computerized patient record system (CPRS) on the same day of the study. Data thus collected included age, Body Mass Index (BMI), Hemoglobin A1C (HbA1C), serum lipids, and the presence of other comorbid diseases. For this study clinically significant apathy was defined as an AES-C score $>30$, which is the typically used cut-off.

\subsection{Statistical analysis}

The data were entered in a database and analyzed using SAS 9.0 statistical software. Characteristics of patients with and without clinically significant apathy, including HbA1c, BMI, cholesterol levels, age, HAM-D scores and SCI scores, were compared using two-sample t-test. Multiple linear regression was used to investigate the association between clinically significant apathy and factors that affect glycemic control, while adjusting for age, BMI, total cholesterol, mild depression (HAM-D $\geq 7$ ) and the average SCI score. Subdomains of apathy including motivation, persistence and novelty were also analyzed similarly. Descriptive summaries are presented as the mean \pm S.D. The significance level was set at 0.05 . 


\subsection{Instruments}

\subsubsection{Apathy Evaluation Scale-Clinician version (AES-C)}

[15]

AES-C has been specifically developed to assess apathy and discriminate it from depression. This 18-item scale with scores ranging from 18 to 72 assesses apathy in behavioral, cognitive and emotional domains over the previous four weeks. Three questions have negative syntax to ensure validity of responses. A semi-structured interview is conducted to obtain information from the patient. The AES-C has good internal consistency (coefficient alpha $=0.86$ ), and test-retest reliability $(r=0.76)$. A score of $>30$ is considered clinically significant apathy [21]. Apathy is divided into three sub-domains of persistence, motivation and novelty since the deficits in each of these sub-domains could differentially impact the outcomes. The sub-domains of apathy were obtained by combining the responses to questions in the AES-C that targeted the specific construct. Persistence domain was derived from responses to questions 9 'Seeing a job through is important to him/her' and 10 'S/he spends time doing things that interest him/her'. Motivation domain consisted of questions 1 ' $\mathrm{S} /$ he is interested in things', 16 'Getting things done during the day is important to him/her' and 18 'S/he has motivation'. Novelty domain was derived using questions 3 'S/ he is interested in having new experiences' and 4 ' $\mathrm{S} / \mathrm{he}$ is interested in learning new things' [21]. The cutoffs for subdomains were obtained using the same model used for the AES-C score adjusted to the number of questions. A score of $\leq 3$ was considered as intact initiative and persistence and a score of $\leq 5$ was considered intact motivation.

\subsubsection{Hamilton Rating Scale for Depression (HAM-D $\left.{ }_{17}\right)$ [16]}

The 17-item version of the HAM-D was used for this study [22]. The HAM- $\mathrm{D}_{17}$ is widely used for rating the severity of the symptoms depression and a score of $>14$ is typically considered as mild to moderate depression. A score of $<7$ is usually considered as being free of depression in clinical trials.

\subsubsection{The Self-Care Inventory (SCI) [17]}

The SCI is a measure of perceptions of adherence to diabetes self-care recommendations. 14 items targeting adherence to various diabetes self-care recommendations over the previous month are scored on a Likert scale ranging from 1 "never do it" to 5 "always do this as recommended without fail". Five items target insulin and food regulation, four items concern blood
Table 1 - Demographics: mean $(\mathrm{N}=\mathbf{8 1})$

\begin{tabular}{ll} 
Age (years) & $58.6( \pm 11.9)$ \\
Male & $94 \%$ \\
Caucasian & $88 \%$ \\
African American & $11 \%$ \\
Hispanic & $1 \%$ \\
Apathy score & $36.17( \pm 11.19)$ \\
HbA1C (\%) & $8.1( \pm 1.7)$ \\
HAM-D & $6.5( \pm 3.7)$ \\
BMI & $32.7( \pm 7.3)$ \\
Total cholesterol $(\mathrm{mg} / \mathrm{dL})$ & $179.9( \pm 49.4)$ \\
\hline
\end{tabular}

glucose regulation, two items target exercise, two target emergency precautions and one item targets attendance to appointments. The SCI has a good internal consistency (coefficient alpha $>0.80)$, and test-retest reliability $(r>0.77)$ [23]. The authors have previously identified 7 of the 14 items to be linked with better diabetes management and control [23]. These items include \#1, 2, 5, 6, 7, 8 and 13; aggregates of these items have been used in our analysis.

\section{Results}

Hundred consecutive patients were enrolled and interviewed, 19 of whom had scores greater than 14 on the HAM-D and were thus excluded from analysis to avoid confounding. Data from the remaining 81 patients was analyzed.

The mean age of the 81 patients was $58.60 \pm 11.99$ years, 76 (94\%) patients were male, 71 (88\%) were Caucasian, 9 (11\%) were African American and 1 patient was Hispanic American. The mean apathy score was $36.17 \pm 11.19$, indicating clinically significant apathy in this population. The mean HAM-D score was $6.52 \pm 3.72$ indicating a lack of clinically significant depression in this population. Thus, the 81 patients included in the analysis had an apathy syndrome without depression. Other demographic data and baseline values are presented in Table 1.

Fifty of the 81 patients (61.7\%) had clinically significant apathy (AES-C > 30). After adjustment for age, BMI, cholesterol, mild depression, and the 7-item diabetes SCI average score, the mean HbA1C levels was $0.66 \%$ higher (absolute difference) for subjects with apathy compared to subjects without apathy, which was not statistically significant $(p=0.08)$, but had a definite trend to being higher.

Patients with apathy had a higher mean BMI (34.07 versus $30.46, p=0.03)$. Patients with apathy were less likely to adhere

\section{Table 2 - Impact of apathy on self-care of diabetes, BMI and the glycemic control}

\begin{tabular}{|c|c|c|c|}
\hline Variable & No Apathy (AES $\leq 30$ ) & Apathy (AES > 30) & $p$-value \\
\hline HbA1C (\%) (multivariate analysis) & $7.64( \pm 1.49)$ & $8.3( \pm 1.55)$ & 0.08 \\
\hline BMI & $30.5( \pm 7.14)$ & $34.1( \pm 7.09)$ & $0.03^{*}$ \\
\hline Adherence to a meal plan ${ }^{a}$ & $3.32( \pm 1.14)$ & $3.02( \pm 1.11)$ & 0.243 \\
\hline Adherence to an exercise plan ${ }^{a}$ & $3.45( \pm 1.48)$ & $2.64( \pm 1.41)$ & $0.019^{*}$ \\
\hline Administering correct dose of insulin ${ }^{\mathrm{a}}$ & $4.88( \pm 0.34)$ & $4.44( \pm 0.61)$ & $0.003^{*}$ \\
\hline Measuring glucose regularly ${ }^{\mathrm{a}}$ & $4.23( \pm 1.05)$ & $3.83( \pm 1.18)$ & 0.132 \\
\hline
\end{tabular}


Table 3 - Impact of sub-domains of apathy on glycemic control

\begin{tabular}{llc} 
Subdomains of Apathy & HbA1c $(\%)$ & $p$-value \\
\hline $\begin{array}{l}\text { Motivation } \\
\text { Intact }(\leq 5)\end{array}$ & $7.74( \pm 1.63)$ & 0.08 \\
Loss of $(>5)$ & $8.37( \pm 1.62)$ & \\
Persistence & & \\
Intact $(\leq 3)$ & $7.83( \pm 1.45)$ & 0.04 \\
Loss of $(>3)$ & $8.53( \pm 1.82)$ & \\
Novelty & & \\
Intact $(\leq 3)$ & $8.0( \pm 1.6)$ & \\
Loss of $(>3)$ & $8.15( \pm 1.72)$ & \\
\hline$p<0.05$. & & \\
\hline
\end{tabular}

to an exercise plan $(p=0.01)$ or to administer the correct doses of insulin ( $p=0.003)$ when compared to those without apathy (Table 2). There were no significant differences in patients with and without apathy in terms of adherence to meal plans or measuring subcutaneous glucose regularly.

We further tested the impact of the sub-domains of apathy on glycemic control. The lack of persistence was significantly associated with poor glycemic control (7.83 versus 8.53, $p=0.04)$, whereas the lack of motivation showed a trend towards association with poor glycemic control (7.74 versus 8.37, $p=0.08$ ) (Table 3). The lack of novelty had no association with poor glycemic control (8.0 versus $8.15, p=0.84$ ).

\section{Discussion}

The primary finding in this study is a high prevalence of apathy (62\% clinically significant apathy) in this cohort of predominantly white, male veteran population with diabetes. This is the first study to the best of our knowledge that assesses the prevalence of apathy in such a population. Generalizability of this data may be limited due to the gender bias; nevertheless the high prevalence of apathy in patients with diabetes is alarming. The mean apathy score was 36.17 despite the mean scores on the HAM-D being 6.52. This indicates that clinically significant apathy can exist in the absence of depression. Similar discordance between apathy and depression has been reported in other chronic illnesses such as dementia [9]. Even more alarming is the fact that apathy was not diagnosed in any of these patients on a clinical basis prior to our study.

The secondary finding of the study shows that factors that are important in glycemic control were significantly affected by apathy. Patients with apathy had significantly higher BMI when compared to those without apathy. Patients with apathy were also less likely to follow an exercise plan or to take their insulin as instructed. Diabetes control itself as measured by HbA1c trended higher $(0.66 \%)$ in patients with apathy but failed to reach statistical significance. Together these suggest that patients with apathy are likely to exhibit behaviors that worsen diabetes control and thus its complications. The fact that these associations may exist in a population devoid of clinically significant depression, suggests that apathy may have a significant role in factors that affect glycemic control. This strengthens our hypothesis that the presence of apathy impairs self-care, thus the treatment of apathy opens another avenue to impact the control of diabetes.

We further tested if the subdomains of apathy, lack of persistence, motivation and initiative were linked to poor glycemic control. Diabetes patients with a lack of persistence and motivation had a higher HbA1c. This further suggests that a lack of persistence and motivation may be major contributors to the inability of some diabetes patients to adhere to self-care behaviors.

The clinical implications of these findings could be substantial. Apathy may be highly prevalent in patients with diabetes. Apathy may be associated with poor control of factors that affect diabetes, weight gain and may often go undiagnosed. Clinicians and educators may want to screen for apathy routinely in clinical care of patients with diabetes, especially in patients with poor control. Although the AES-C is an excellent rating scale for apathy, the time needed to complete this scale may hinder practical use of this rating scale in routine clinical practice. The patient/caregiver rated versions of AES may help detect apathy reliably without added burden on the physician. Another useful screen in busy clinical practice are the four screening questions from the apathy domain of the Neuropsychiatric Inventory (NPI). 'Has the patient lost interest in the world around him/her?', 'Has he/she lost interest in doing things or lacks motivation for starting new activities?', 'Is he/she more difficult to engage in conversation or in doing chores?', and 'Is the patient apathetic or indifferent?' [24]. More detailed testing will be needed if the patient endorses any of the screening questions.

Several agents have been used for the treatment of apathy, including methylphenidate, cholinesterase inhibitors, bupropion, bromocriptine, and modafinil [25-29]. Large randomized trials testing these agents are however lacking. Although it is plausible that self-care behaviors and factors that are important for diabetes control, could improve with successful treatment of apathy, it needs to be tested in systematic studies.

Limitations of this study include but are not limited to its cross-sectional design. Prospective studies, including the assessment of apathy at the time of the initial diagnosis of diabetes, and periodic re-assessments of apathy over a period of a few years is necessary to establish any causal relation. Comorbid conditions were not accounted for systematically, use of a risk adjustment measure such as the Charlson Index could improve the methodology of future studies. $94 \%$ of the patients in this study were male as it was conducted in the VA, it will be interesting to investigate gender differences in future studies.

In conclusion, our study in the predominantly male veteran population, found a high prevalence of apathy in patients with diabetes. Patients with apathy were less likely to adhere to self-care behaviors that influence diabetes control and had a higher BMI. Diabetes control itself as measured by HbA1c trended higher in patients with apathy but failed to reach statistical significance.

\section{Acknowledgments}

Robert Marin, MD, Associate Professor, Department of Psychiatry, University of Pittsburgh Medical Center, for 
training Dr. Padala in administration of the Apathy Evaluation Scale.

Annette M. La Greca, PhD, Professor, Child Division, Department of Psychology, University of Miami, for sharing the Self Care Inventory with Dr. Padala and permitting its use in this study.

Julie Stoner, PhD, Associate Professor, Department of biostatistics, College of Public Health, Omaha, NE for her help in the data analysis.

\section{R E F E R E N C E S}

[1] J.S. Skyler, C. Oddo, Diabetes trends in the USA, Diab. Metab. Res. Rev. 18 (Suppl. 3) (2002) S21-S26.

[2] D.R. Miller, M.M. Safford, L.M. Pogach, Who has diabetes? Best estimates of diabetes prevalence in the Department of Veterans Affairs based on computerized patient data, Diab. Care 27 (Suppl. 2) (2004) B10-B21.

[3] G.E. Reiber, E.J. Boyko, C. Maynard, T.D. Koepsell, L.M. Pogach, Diabetes in the Department of Veterans Affairs, Diab. Care 27 (Suppl. 2) (2004) B1-B2.

[4] M.I. Harris, Diabetes in America: epidemiology and scope of the problem, Diab. Care 21 (Suppl. 3) (1998) C11-C14.

[5] P. Hogan, T. Dall, P. Nikolov, American Diabetes Association. Economic costs of diabetes in the US in 2002, Diab. Care 26 (3) (2003) 917-932.

[6] S.H. Saydah, J. Fradkin, C.C. Cowie, Poor control of risk factors for vascular disease among adults with previously diagnosed diabetes, JAMA 291 (2004) 335-342.

[7] D.L. Evans, D.S. Charney, L. Lewis, Mood disorders in the medically ill: scientific review and recommendations, Biol. Psychiatry 58 (3) (2005) 175-189.

[8] L.C. Brown, S.R. Majumdar, S.C. Newman, J.A. Johnson, History of depression increases risk of type 2 diabetes in younger adults, Diab. Care 28 (5) (2005) 1063-1067.

[9] M. de Groot, R. Anderson, K.E. Freedland, R.E. Clouse, P.J. Lustman, Association of depression and diabetes complications: a meta-analysis, Psychosom. Med. 63 (4) (2001) 619-630.

[10] P.K. Singh, H.C. Looker, R.L. Hanson, J. Krakoff, P.H. Bennett, W.C. Knowler, Depression, diabetes, and glycemic control in Pima Indians, Diab. Care 27 (2) (2004) 618-619.

[11] R. Gross, M. Olfson, M.J. Gameroff, O. Carasquillo, S. Shea, A. Feder, R. Lantigua, M. Fuentes, M.M. Weissman, Depression and glycemic control in Hispanic primary care patients with diabetes, J. Gen. Intern. Med. 20 (5) (2005) 460-466.

[12] P.M. Trief, P.C. Morin, R. Izquierdo, J. Teresi, J.P. Eimicke, R. Goland, J. Starren, S. Shea, R.S. Weinstock, Depression and glycemic control in elderly ethnically diverse patients with diabetes: the IDEATel project, Diab. Care 29 (4) (2006) 830-835.
[13] M.L. Levy, J.L. Cummings, L.A. Fairbanks, et al., Apathy is not depression, J. Neuropsychiatry Clin. Neurosci. 10 (3 (Summer)) (1998) 314-319.

[14] R. van Reekum, D.T. Stuss, L. Ostrander, Apathy: why care? J. Neuropsychiatry Clin. Neurosci. 17 (1) (2005) 7-19.

[15] R.S. Marin, Apathy: a neuropsychiatric syndrome, J. Neuropsychiatry Clin. Neurosci. 3 (3) (1991) 243-254.

[16] R.S. Marin, Differential diagnosis and classification of apathy, Am. J. Psychiatry 147 (1) (1990) 22-30.

[17] P. Thomas, J.P. Clement, C. Hazif-Thomas, J.M. Leger, Family Alzheimer's disease and negative symptoms, Int. J. Geriatr. Psychiatry 16 (2) (2001) 192-202.

[18] S. Freels, D. Cohen, C. Eisdorfer, G. Paveza, P. Gorelick, D.J. Luchins, et al., Functional status and clinical findings in patients with Alzheimer's, J. Gerontol. 47 (6) (1992) M177M182.

[19] V.L. Lawson, P.A. Lyne, J.N. Harvey, et al., Understanding why people with type 1 diabetes do not attend for specialist advice: a qualitative analysis of the views of people with insulin-dependent diabetes who do not attend diabetes clinic, J. Health Psychol. 10 (3) (2005) 409-423.

[20] B.D. Puente, K.K. Nichols, Patients' perspectives on noncompliance with diabetic retinopathy standard of care guidelines, Optometry 75 (11) (2004) 709-716.

[21] R.S. Marin, R.C. Biedrzycki, S. Firinciogullari, Reliability and validity of the Apathy Evaluation Scale, Psychiatry Res. 38 (2) (1991) 143-162.

[22] M. Hamilton, A rating scale for depression, Neurol. Neurosurg. Psychiatry 23 (1960) 56-62.

[23] A.M. La Greca, Brief Manual for the Self-Care Inventory, Miami, Fl, 1992 (unpublished data).

[24] J.L. Cummings, M. Mega, K. Gray, S. Rosenberg-Thompson, D.A. Carusi, J. Gornbein, The Neuropsychiatric Inventory: comprehensive assessment of psychopathology in dementia, Neurology 44 (12) (1994) 2308-2314.

[25] A. Chatterjee, S. Fahn, Methylphenidate treats apathy in Parkinson's disease, J. Neuropsychiatry Clin. Neurosci. 14 (4) (2002) 461-462.

[26] J.L. Cummings, B. Koumaras, M. Chen, D. Mirski, Rivastigmine Nursing Home Study Team. Effects of rivastigmine treatment on the neuropsychiatric and behavioral disturbances of nursing home residents with moderate to severe probable Alzheimer's disease: a 26week, multicenter, open-label study, Am. J. Geriatr. Pharmacother. 3 (3) (2005) 137-148.

[27] C. Corcoran, M.L. Wong, V. O'Keane, Bupropion in the management of apathy, J. Psychopharmacol. 18 (1) (2004) 133-135.

[28] C.E. Catsman-Berrevoets, F. von Harskamp, Compulsive pre-sleep behavior and apathy due to bilateral thalamic stroke: response to bromocriptine, Neurology 38 (4) (1988) 647-649.

[29] P.R. Padala, W.J. Burke, S.C. Bhatia, Modafinil therapy for apathy in an elderly patient, Ann. Pharmacother. 41 (2) (2007) 346-349. 